\title{
EL PRODUCTO INDUSTRIAL DE POSGUERRA: UNA REVISION (Indices sectoriales, 1940-1958)*
}

ENRIC MORELLÁ Universidad Jaime I

\section{RESUMEN}

El trabajo analiza la evolución de la producción industrial española en el período comprendido entre la Guerra Civil y el Plan de Estabilización, con especial referencia a la década de los cuarenta.

Para ello se lleva a cabo una revisión del IPIES propuesto por Albert Carreras para mejorar su cobertura. Esta reelaboración del índice de la producción industrial se hace de forma desagregada, de manera que sea posible obtener un índice para cada sector y captar así los cambios en la estructura productiva de la industria española en el período.

\section{ABSTRACT}

This paper analyzes the Spanish industrial product during the period between the Spanish Civil War and the stabilization plan of 1959, whith special attention to the 1940's.

The essay carries out a revision of the Industrial Production Index, developed by Albert Carreras. The new industrial production index is made by breaking down the aggregate, in such a way that an index for each sector can be compiled, allowing for the changes in the productive structure of the Spanish industry of the period.

La información cuantitativa usada por los historiadores económicos para calibrar el proceso industrializador de España en el presente siglo provenia, hasta la publicación del indice de la producción industrial del profesor

* Debo agradecer la colaboración de J. Palafox, quien, al margen de su constante estímulo en la investigación, tuvo la paciencia de revisar diferentes versiones de este texto. C. Betrán, $\mathrm{S}$. Calatayud, A. Cubel, M. A. Pons, D. Tirado y E. Verdeguer aportaron valiosas sugerencias en sucesivas discusiones del trabajo. Los etrores que subsisten son, pues, de mi responsabilidad. 
Carreras ${ }^{1}$, de las series del Consejo de Economía Nacional y del Instituto Nacional de Estadística ${ }^{2}$. La visión que resultaba era la de un crecimiento continuo a lo largo del siglo, protagonizado por la industria pesada y con una inequívoca aceleración a partir de 1950. Con mayores o menores reservas, dadas las ineficiencias en la asignación de recursos, existía un cierto consenso en afirmar que con las medidas protectoras, fomentadoras, interventoras y autárquicas que se sucedieron, la industrialización española contemporánea se había desarrollado hasta $1960^{3}$.

Los nuevos datos aportados por Carreras muestran con claridad la discontinuidad que representa la guerra civil. En los índices anteriores, los niveles máximos de preguerra se volvían a alcanzar en 1942. Según el IPIES, en cambio, el crecimiento de posguerra no se inicia hasta 1950. Así, tras el período $1935-40$ en que el producto industrial se derrumba, la recuperación es lenta ${ }^{4}$.

Este trabajo pretende avanzar un paso más en el análisis de los avatares de la industria española en la posguerra. Se parte de la convicción de que ni los niveles de producto industrial ni las tasas de crecimiento de esta magnitud agregada, con constituir una información valiosa, son suficientes para conocer la senda seguida por una economía en el proceso de industrialización. Un comportamiento perezoso del agregado puede estar escondiendo profundos reajustes internos, cuyo conocimiento es necesario para valorar justamente tanto sus logros o fracasos como la responsabilidad de la política económica.

1 Carreras (1984). En adelante, para referimos a este indice, utilizaremos la abreviatura IPIES, propuesta por el propio autor y ya conocida en la literatura.

${ }^{2}$ C. E. N. (1965); I. N. E. (1982). Otras estimaciones no oficiales están descritas en I. N. E. (1982). Cabe destacar la de J. Alcaide, cuyas deficiencias están expuestas en la tesis de licenciatura de $A$. Carreras, desgraciadamente inédita. Una breve reseña de estas objeciones se puede hallar en Carreras (1984), p. 129.

3 El ejemplo más claro es Paris Eguilaz a lo largo de toda su obra. Pero también, más recientemente y desde otra perspectiva, Braña, Buesa y Molero (1984), p. 200, cuando señalan que «lo realizado en los dos primeros decenios del franquismo sentó las bases de la estructura productiva que, después de 1960 , se desarrollaria ampliamente, en una coyuntura internacional más favorable», o cuando tachan de precipitadas y faltas de análisis las conclusiones que conllevan una valoración negativa de la política industrial en el período autárquico.

4 Según la explicación ofrecida por el propio Carreras, las series oficiales muestran mayor elasticidad de recuperación tras la guerra civil por el excesivo peso otorgado a la industria pesada. Ello conduce a pensar en un comportamiento diferenciado de los distintos sectores. Precisamente estos cambios en la estructura productiva de la industria son los que aquí se investigan.

También hay problemas técnicos en el origen de las discrepancias entre series. En concreto, el sesgo al alza de los indices oficiales por el hecho de ponderar con precios de 1929. Es lo que en la literatura sobre números índices se conoce como efecto Gerschenkron [véase Scott (1952)]: las tasas de crecimiento son tanto más bajas cuanto más próximos a nosotros son los precios usados para ponderar, y viceversa. 
Ello es así porque la composición del producto no es neutral de cara al crecimiento. Basta recordar que un aumento en los bienes de inversión no sólo repercute en el producto corriente, sino también en la capacidad productiva de la economía. Por tanto, con un estudio desagregado de la industria española en la posguerra no sólo nos acercaremos más a la realidad del período, sino que también estaremos mejor armados para entender el acelerado crecimiento que siguió al Plan de Estabilización.

El objetivo de estas páginas es presentar la revisión del IPIES, llevada a cabo como medio de evaluar la composición del producto industrial entre 1940 y 1958; el cambio estructural, en definitiva. En la primera parte se explicitan los criterios y métodos seguidos. Los detalles más técnicos en la construcción del nuevo índice son explicados en un apéndice al fonal del trabajo. En la segunda parte se analiza el comportamiento desagregado de la industria española en la posguerra, en conexión con las directrices de política económica, y se discuten sus implicaciones para el desarrollo económico. Finalmente, dado que este trabajo conlleva la propuesta de un nuevo índice (IPIE-PGC), se estudian brevemente sus rasgos diferenciales con el IPIES.

Hay dos cuestiones de método que, de entrada, es preciso hacer notar. La primera es que se ha rehusado la revisión de las series de producción fisica utilizadas por Carreras. La razón fundamental es que, aunque para la construcción de un índice largo como el IPIES se hubo de recurrir a la reelaboración de series dada la ausencia de homogeneidad en los datos ${ }^{5}$, para el período aquí considerado la mayor parte de la información proviene de series originales $y$, por tanto, no admite otra discusión que la credibilidad de sus fuentes. Además, la tarea de revisar exhaustivamente los datos primarios es de tal magnitud que excede los límites razonables en un trabajo de este tipo.

El segundo punto se refiere a los sistemas de ponderación. El que más se acerca al ideal es el de valores añadidos unitarios variables anualmente ${ }^{6}$. Sin embargo, la escasa información disponible sobre los precios industriales de

5 Aunque los criterios para la reelaboración de series siempre pueden ser discutidos, en Carreras (1983) éstos se hallan suficientemente explicitados, con lo que cualquier investigador puede analizarlos.

- Para mayor información sobre números indices, puede verse Usher (1980). 
posguerra impide utilizarlo?. La estimación que se presenta sigue, pues, un sistema de ponderación fija similar al del IPIES, basado en los valores añadidos por unidad de producto del año final ${ }^{8}$.

Cabe concluir, por tanto, que esta revisión no cuestiona, al menos como criterio general, ni las fuentes de información ni la fundamentación teórica necesarias para la elaboración de un indice de la producción industrial. Se trata de adaptar un indice largo como es el IPIES, que cubre ciento cuarenta años, al período 1940-58. Ello implica corregir algunas ponderaciones, incluir series que fueron omitidas en aras a la continuidad del indice y prescindir, en otros casos, de las irrelevantes para este período concreto. Con todo, el interés de la tarea va más allá de la mera adaptación del índice a un período corto. Quizá su mayor mérito consista en que, al ser desagregado en los grandes sectores más significativos, permite conocer no sólo el ritmo de industrialización, sino también la composición del producto?

II

En el cuadro número 1 puede verse la evolución de la producción industrial por sectores expresada en tasas anuales de crecimiento. Un primer rasgo a destacar es lo elevado de éstas en el sector energético, a pesar de las fuertes restricciones en el suministro eléctrico (con diferencia, el producto con más peso en el sector), especialmente en los años cuarenta ${ }^{10}$. El bajo nivel de partida y la intensidad del proceso de sustitución, tanto en el consumo como en la producción, en favor de la energía eléctrica parecen suficientes para explicar este dinámico comportamiento a pesar de que se mantuvieran insatisfechas las necesidades de la demanda.

\footnotetext{
7 Se suele recurrir a los precios como proxy, dado que para series históricas normalmente es imposible estimar directamente el valor añadido. Sin embargo, sólo a partir de 1955 disponemos de información aceptable sobre precios debida al I. N. E. En Carreras (1983), pp. 685-698, se ofrece una excelente panorámica de la información disponible.

${ }^{8}$ La justificación teórica se halla en Fenoaltea (1976). Las ponderaciones proceden de la tabla input-output española de 1958, TIOE-58 (1962). Aún disponemos de una TIOE anterior, la de 1954, pero su excesivo grado de agregación impide enriquecer el índice con esta información.

9 Las únicas noticias sobre el comportamiento sectorial para el período de posguerra se derivan de una desagregación llevada a cabo por Carreras, pero a partir de un indice que toma como años de ponderación 1970 [Carreras (1983), pp. 1049-1050]. La magnitud del cambio estructural operado en España desde la guerra civil hasta la crisis de los setenta y las consiguientes alteraciones en los precios relativos aconsejan tomar estos datos con mucha prudencia.
}

10 Véase Sudrià (1987), pp. 332-335. 
CUADRO 1

Tasas de crecimiento

\begin{tabular}{|c|c|c|c|c|c|c|c|}
\hline \multirow{2}{*}{\multicolumn{2}{|c|}{ Periodo }} & \multicolumn{6}{|c|}{ Tasas medias de crecimiento anual acumulativo } \\
\hline & & 1 & $I I$ & $I I I$ & $1 V$ & $V$ & $I P I E-P G C$ \\
\hline $1940-58$ & $\ldots$ & 6,2 & 4,8 & 10,7 & 5,5 & 3,5 & 4,7 \\
\hline $1940-50$ & & 4,4 & 2,2 & 10,4 & 1,9 & 0,8 & 2,2 \\
\hline $1950-58$ & & 8,6 & 8,1 & 11,2 & 10,1 & 6,9 & 7,9 \\
\hline $1940-45$ & & 3,8 & $-2,7$ & 3,2 & $-3,6$ & 2,1 & 0,8 \\
\hline $1945-50$ & $\ldots \ldots \ldots \ldots \ldots \ldots$ & 5,0 & 7,4 & 18,0 & 7,7 & $-0,5$ & 3,6 \\
\hline $1950-55$ & $\ldots \ldots \ldots \ldots \ldots \ldots$ & 7,4 & 8,2 & 12,7 & 9,0 & 7,3 & $.7,7$ \\
\hline $1955-58$ & 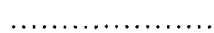 & 10,5 & 8,0 & 8,7 & 11,9 & 6,1 & 8,2 \\
\hline
\end{tabular}

1, Energía. II, Minería y primera transformación. III, Química. IV, Transformación de metales. $\mathrm{V}$, Industrias de consumo.

FUENTE: Elaboración propia a partir de la tabla número 1.

Conviene tener presente, sin embargo, que casi la mitad del producto energético viene explicado por la electricidad, ya que este hecho disminuye la calidad de la estimación. Un sistema de ponderación fija como el adoptado ignora los cambios en los precios relativos que van indisolublemente unidos a los cambios en la composición del producto. El hecho de que la electricidad viera descender su precio relativo un 64 por 100 en el período, aconseja tratar de utilizar una ponderación variable, al menos en este sector ${ }^{11}$.

Además, en el caso de la energía, con sólo dos productos (hulla y electricidad) se alcanza un grado de cobertura suficientemente alto (67 por 100) como para considerarlos representativos. Ello simplifica los cálculos necesarios, dado que la utilización de ponderaciones variables requiere la normalización de los valores añadidos unitarios respecto al año base ${ }^{12}$.

11 Lo peculiar del comportamiento del precio relativo de la electricidad es analizado en Carreras (1984), p. 133-5. La caída de su precio relativo se ha calculado con los datos sobre la evolución del Indice de Precios al por Mayor (ponderado) [Carreras (1989, a), Pp. 521-2] y del precio de la electricidad [Carreras $(1989$, a), p. 218].

12 La normalización implica resolver, para todo $i$, la siguiente ecuación:

$$
p_{i 1} q_{11}+p_{i 2} q_{12}=p_{11} q_{i 1}+p_{t 2} q_{12}
$$

donde debe cumplirse la condición de que el ratio entre $p_{i 1} y p_{i 2}$ sea igual al precio relativo entre ambos productos en el año correspondiente. 
Como se deduce del cuadro número 2, la toma en consideración de los cambios en los precios relativos ofrece una visión más dinámica del comportamiento de la energía en la posguerra, ya que eleva un 30 por 100 la tasa media de crecimiento anual. Como era previsible, la ponderación fija en 1958 subestimaba el auge del sector, porque asignaba a la electricidad un peso demasiado bajo, dado que su ponderación era evaluada al final de un período en que decrece su precio relativo. Si se analiza el cuadro número 2 con mayor detenimiento, se puede constatar cómo el uso de ponderaciones variables multiplica por 2,3 el crecimiento medio anual de la segunda mitad de los

\section{CUADRO 2}

La producción energética de posguerra (tasas de crecimiento)

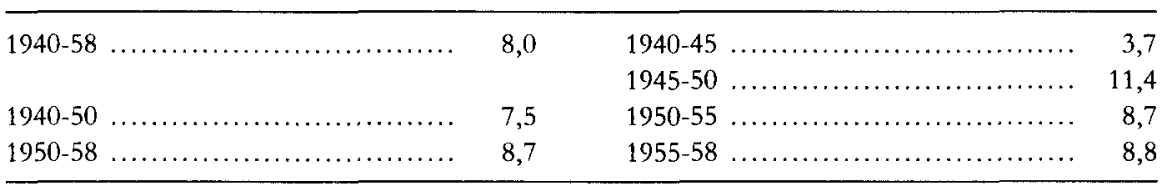

FUENTE: Elaboración propia. Las tasas proceden del índice energético con ponderaciones variables al que se refieren el texto y la nota número 12 .

cuarenta, lo cual es indicativo de que el cambio estructural fue especialmente intenso en este período. Este hecho se demuestra con mayor contundencia en páginas posteriores.

En la industria química no parece que operaran tales restricciones por el lado de la oferta, puesto que el sector experimentó en la segunda mitad de la década de los cuarenta un progreso espectacular. Seguramente, el tirón de la demanda internacional en la segunda guerra mundial y los procesos de recuperación subsiguientes explican este comportamiento. De hecho, en los años cincuenta, cuando los intercambios internacionales se normalizan, el sector químico desaceleró su crecimiento de forma acusada.

La industria de bienes de consumo también se aleja de la media industrial, pero por defecto. El estado de los conocimientos en la historiografia española sobre la posguerra, aunque insuficiente, arroja algunos elementos para explicar esta evolución. Lo primero que llama la atención en el índice es que este

El precio relativo entre la hulla y la electricidad procede de Carreras (1989, a), p. 218 , columnas 3 y 6 . Las cantidades $q_{11}$ y $q_{12}$ y los valores añadidos unitarios $p_{t 1}$ y $p_{t 2}$ son los mismos que han servido para la construcción del IPIE-PGC (véase fuentes de la tabla n. 1). 
sector no parece haber sido el más perjudicado en la inmediata posguerra. Al contrario, es el sector de bienes de equipo el que presenta tasas de crecimiento negativas para la primera mitad de la década de los cuarenta. Esto se explica por el nivel extraordinariamente bajo de las industrias de consumo tras la guerra civil (véase cuadros números 6 y 7). Como apunta Farré-Escofet:

los fenómenos característicos posteriores al desastre de la guerra son la escasez (...) y la falta de recursos o de capacidad de ahorro ${ }^{13}$.

En cambio, es en la segunda mitad de la década cuando el índice evidencia una caida de las industrias productoras de bienes de consumo, con tasas de crecimiento negativas, en el marco de una coyuntura general bastante expansiva, con una fuerte recuperación de la siderometalurgia y la industria mecánica y con el despegue de la química antes mencionado. También el sector energético crece por encima de la media. Obviamente, dado el gran peso que todavía tiene en la economía la industria alimentaria y la textil, las tasas de crecimiento del IPIE-PGC son mucho más modestas.

Nos encontramos aquí, con la mayor crudeza, el resultado de una política intervencionista que con una regulación integral de la economía a través, de manera preferente, de un estricto control de precios y salarios, trata de fomentar la industrialización a toda costa. El cambio hacia una estructura interindustrial más moderna se lleva a cabo, pues, con un fuerte constreñimiento del consumo y a costa de una regresiva distribución de la renta.

En palabras de A. Carreras:

La reducción del salario real después de la guerra civil significó, grosso modo, el retorno a la situación retributiva anterior a la primera guerra mundial, tanto en el mundo urbano como en el rural. Sin lugar a dudas, se trata del rasgo más importante de la vida económica y social de la España de la inmediata posguerra, y condicionó fuertemente el resto de variables económicas ${ }^{14}$.

El cuadro número 3 ofrece una información reveladora a este respecto. La caída de la industria de bienes de consumo en la segunda mitad de los cuarenta se explica en su totalidad por la industria textil. Tanto el subsector alimentario como las otras industrias manufactureras mantienen tasas de aumento positivas. Ello pone de relieve la estrecha dependencia de la primera respecto al salario real para bajos niveles de ingreso. La mayor elasticidad renta de la demanda textil respecto a la alimentaria opera para que sea aquélla

\footnotetext{
13 Farré-Escofet (1980), p. 1.

14 Carreras (1989, b), p. 15.
} 
y no ésta la que reciba con más intensidad los efectos de la caída de los salarios reales. Nos encontramos, pues, en pleno siglo XX, ante un fenómeno asimilable a las tijeras en los precios relativos características de las economías preindustriales, lo cual, además, no hace más que subrayar la brutalidad de la contracción del ingreso real disponible para un amplio segmento de la población.

\section{CUADRO 3}

Industria de bienes de consumo (tasas de crecimiento)

\begin{tabular}{|c|c|c|c|}
\hline & Alimentación & Textil & Otras \\
\hline $1940-45$ & 1,12 & 3,65 & 1,21 \\
\hline $1945-50$ & 1,51 & $-3,32$ & 4,76 \\
\hline $1950-55$ & $2,67^{\circ}$ & 7,52 & 12,86 \\
\hline $1955-58$ & 4,73 & 6,09 & 8,05 \\
\hline
\end{tabular}

Fuente: Elaboración propia a partir de la tabla número 1.

A partir de comienzos de la década de los cincuenta, la evolución de este tipo de industrias es bien distinta. Sus tasas se aproximan a la media industrial, aunque siguen siendo las de más lento crecimiento en todo el período. La tendencia del sector de consumo a perder peso en la economía conforme avanza el proceso de industrialización es un fenómeno universal y bien conocido. Esto permite plantear la cuestión del cambio interindustrial en términos de la hipótesis de Hoffmann.

Según su esquema ya tradicional ${ }^{15}$, el desarrollo de las economías hacia la industrialización sigue siempre una pauta uniforme: primero se desarrollan las industrias de bienes de consumo y después las de bienes de equipo. De forma que estas últimas, con el progreso industrial, crecen más rápidamente hasta superar la aportación al producto de las primeras. A partir de esta hipótesis, Hoffman propone su conocida tipología de la industrialización, en la cual, para que un país alcance la madurez industrial, el ratio entre los productos netos de la industria de bienes de consumo y la de bienes de capital ha de estar por debajo de 1,5 .

A. Carreras reprodujo estos cálculos para España e Italia. Según sus resultados, España no podía considerarse un país industrializado en el sentido definido por Hoffmann hasta la década de los años sesenta, mientras que

${ }^{15}$ Hoffman (1958). Es la versión inglesa, revisada y ampliada, del original alemán de 1931. 


\section{GRAFICO 1}

\section{Ratio de Hoffmann}

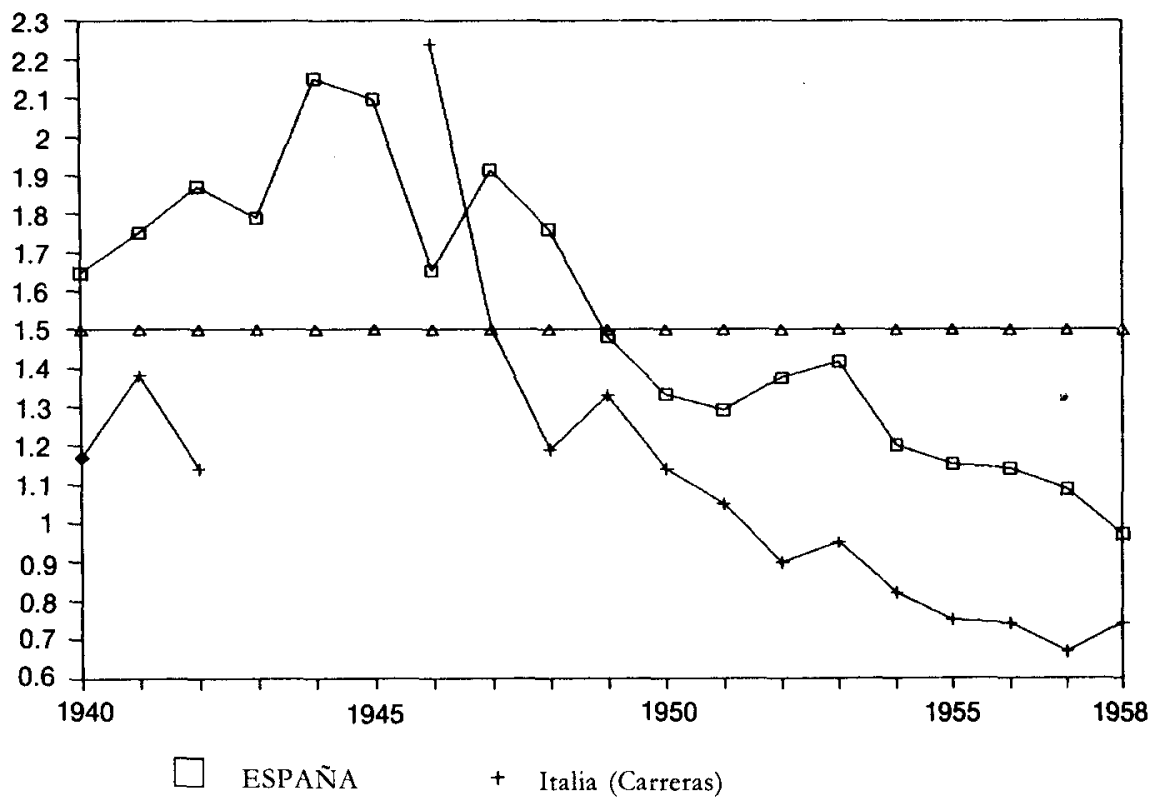

FuENTE: Elaboración según texto y Carreras (83).

Italia ya lo era unos veinte años antes ${ }^{16}$. El resultado es sorprendente, ya que las diferencias en las tasas de crecimiento industrial entre España e Italia no justifican tal disparidad en la velocidad del cambio intraindustrial ${ }^{17}$.

Los índices desagregados de la producción industrial española de posguerra permiten reelaborar el coeficiente de Hoffmann siguiendo fielmente sus criterios de clasificación. Asi, en las industrias de bienes de consumo consideramos las alimentarias, las textiles y las del cuero y los muebles, y en las de bienes de inversión, la construcción de vehículos y la mecánica (en nuestro caso transformados metálicos), la química y la metalurgia ${ }^{18}$. El resultado de esta revisión puede verse en la gráfica número 1.

16 Carreras (1983), pp. 1054-1071.

17 En Carreras (1983), p. 1027, puede hallarse estos datos en tasas de crecimiento anual por decenios desde 1861-1871 a 1971-1980. Con esta información, y suponiendo el mismo nivel de partida para ambos países (lo cual actúa en contra), el producto industrial alcanzado por España en 1961 habría sido logrado en Italia sólo seis años antes.

18 Dado que esta clasificación no coincide con la seguida para construir los índices sectorales, 
El ratio de Hoffman viene a ofrecer, en definitiva, una medida del cambio estructural. De nuevo constatamos un resultado, poco subrayado en la literatura, que ya había sido advertido más arriba. El cambio estructural de mayor envergadura en el período autárquico no se produce en España coincidiendo con las mayores tasas de crecimiento global del producto industrial. De hecho, es en la segunda mitad de los años cuarenta cuando la estructura productiva de la industria española parece aproximarse con mayor rapidez a la de otros países europeos que, como Italia, iniciaron su carrera industrial en la segunda mitad del siglo XIX. En cambio, en los cincuenta la caida en el ratio de Hoffmann se desacelera y la distancia con Italia incluso aumenta ligeramente.

III

Aunque el objetivo del trabajo se centra en la obtención de índices sectoriales para la producción industrial de posguerra, ya que ello supone ampliar la información cuantitativa al alcance de los historiadores económicos que se acerquen a este período, su agregación en el IPIE-PGC viene a ofrecer una estimación del comportamiento de la industria que no puede evitar la comparación con otra ya disponible como el IPIES.

El cuadro número 4 compara las tasas de crecimiento del IPIE-PGC con las del IPIES. Es tranquilizador comprobar cómo las discrepancias no son en absoluto acusadas. Sin embargo, dos rasgos se apuntan con claridad: primero, que el IPIES crece por encima del IPIE-PGC tanto en los años cuarenta como en los cincuenta, y en segundo lugar, que lo anterior deja de ser cierto para la inmediata posguerra.

Estas diferencias se explican por los métodos distintos seguidos en la construcción de uno y otro. El IPIES está concebido como índice agregado, de manera que la producción industrial de cada año se obtiene tras convertir las cantidades de los 107 bienes considerados a producto neto, usando los valores añadidos unitarios como factores de ponderación. El índice resultante será tanto más representativo cuanto más se acerque la gama de productos incluidos a la estructura real de la industria. Sin embargo, con información limitada no suele ser posible satisfacer esta condición, de forma que la

se ha llevado a cabo una desagregación del IPIE-PGC a propósito. Esto significa que se dispone de una estimación separada para la minería y la metalurgia, aunque en el trabajo se presenten ambas ramas conjuntas. 
CUADRO 4

Tasas de crecimiento del IPIES e IPIE-PGC

\begin{tabular}{|c|c|c|}
\hline & IPIES & IPIE-PGC \\
\hline $1940-1958$ & 5,1 & 4,7 \\
\hline $1940-1950$ & 2,4 & 2,2 \\
\hline $1950-1958$ & 8,4 & 7,9 \\
\hline $1940-1945$ & 0,7 & 0,8 \\
\hline $1945-1950$ & 4,2 & 3,6 \\
\hline $1950-1955$ & 7,9 & 7,7 \\
\hline $1955-1958$ & 9,4 & 8,2 \\
\hline
\end{tabular}

FUENTE: Elaboración propia a partir de Carreras (1984), páginas 151-152, y cuadro número 1.

composición del índice viene determinada por la disponibilidad de los datos. Por un lado, nos encontramos lagunas casi insalvables en algunos sectores como el de bienes de consumo ${ }^{19}$, mientras que por el otro, la solución de ignorar información en aquellos sectores, como el energético, donde el grado de conocimiento es mayor, sería suicida. En otras palabras, igualar al alza los grados de cobertura por sectores no es posible en el estado actual de los conocimientos, mientras que igualarlos a la baja es un lujo que no puede permitirse el investigador, cuyo mayor obstáculo suele ser la escasez de información. A. Carreras tuvo en cuenta el problema de la representatividad al construir el IPIES y trató de corregirlo sobrestimando las ponderaciones de aquellos productos que pudieran estar más fuertemente relacionados con el crecimiento industrial agregado, como es el caso de determinados bienes intermedios.

El hecho de disponer de índices desagregados por sectores permite corregir los problemas debidos a la falta de representatividad de un modo menos laborioso y presumiblemente más eficaz. El IPIE-PGC se construye como la media de los índices sectoriales ponderada por la aportación de cada uno de ellos al valor añadido industrial en el año base, para el que la TIOE-58 ofrece toda la información necesaria. De esta forma se hace coincidir la composición del índice con la estructura real de la industria. Bien es cierto que esto sólo se cumplirá exactamente en el año base, pero se puede demostrar que el IPIE-PGC reproduce la estructura industrial en todo el

19 Aunque, según se ha visto en páginas anteriores, en este punto el IPIES mejora sensiblemente la cobertura respecto a los índices oficiales. 
período si el grado de cobertura para cada sector se mantiene estable ${ }^{20}$. Con esta forma de proceder ya no importa que la selección de productos no sea representativa de la composición real de la industria; basta con que lo sean los productos incluidos en cada índice sectorial respecto de su propio sector, lo cual es, sin duda, un supuesto menos fuerte que el anterior.

Ahora estamos en condiciones de explicar las divergencias entre el IPIES y el IPIE-PGC que se apuntaban más arriba. Las tasas de crecimiento del producto industrial que se deducen del IPIES son más elevadas que las del IPIE-PGC, porque algunos de los sectores más dinámicos, como el energético o el de transformados metálicos, estaban representados en exceso. No es sorprendente que para la primera mitad de los cuarenta no se cumpla lo anterior. El ritmo de crecimiento evidenciado por el IPIE-PGC supera ligeramente al del IPIES precisamente porque en este período el cambio estructural es regresivo (tramo creciente del ratio de Hoffmann). Es decir, las industrias pesadas, que son las más representadas, crecen por debajo de la media.

El nuevo índice también es útil para evaluar la magnitud de la caída del producto industrial tras la guerra civil (cuadro n. 5). Mientras el IPIES observa una disminución del 14 por 100 acumulada en cinco años, el IPIEPGC eleva esta caída al 20 por 100. El cuadro número 6 refleja cómo es la industria ligera la que explica la mayor parte de la contracción de la inmediata posguerra. El resto de sectores, salvo la quimica, explican en 1940 un producto superior al de antes de la contienda. De ahí que el IPIES subestime el impacto de la guerra civil, ya que precisamente las industrias de consumo son las más desconocidas $y$, por tanto, el índice no valora suficientemente su verdadero peso en la economía.

\section{CUADRO 5}

Indices del Producto Industrial antes y después de la Guerra Civil $(1958=100)$

\begin{tabular}{|c|c|c|}
\hline & IPIES & $I P I E-P G C$ \\
\hline 1935 & 47,9 & 55,1 \\
\hline $1940 \quad \ldots \ldots \ldots \ldots \ldots$ & 41,1 & 43,8 \\
\hline
\end{tabular}

FUENTE: Carreras (1984), pp. 151-152, y elaboración propia.

20 En realidad, el IPIE-PGC se ha construido tal como se explica en el apéndice. Es decir, sumando para cada año los valores añadidos estimados para los diferentes sectores con el supuesto de que los grados de cobertura se mantienen constantes. Sin embargo, en la nota número 22 se demuestra que ambas formas de proceder son equivalentes. 
CUADRO 6

Producto Industrial por sectores antes y después de la Guerra Civil $(1958=100)$

\begin{tabular}{lllllll}
\hline & & $I$ & $I I$ & II & IV & V \\
\cline { 2 - 6 } 1935 & $\ldots \ldots \ldots \ldots \ldots \ldots \ldots \ldots \ldots \ldots$ & 27,5 & 42,9 & 23,4 & 35,9 & 80,9 \\
$1940 \ldots \ldots \ldots \ldots \ldots \ldots \ldots \ldots \ldots$ & 33,7 & 43,1 & 16,0 & 38,5 & 54,2 \\
\hline
\end{tabular}

FUENTE: Elaboración propia.

Como se deduce del cuadro número 7 , la guerra supuso una clara contracción en todos los subsectores de consumo. El caso más llamativo es el de la alimentación, bebidas y tabaco, que en 1958 todavía no había recuperado su nivel de preguerra. Este hecho confirma el interés por investigar cómo las condiciones imperantes en la posguerra $y$, sobre todo, los criterios de política industrial seguidos, determinaron tal reasignación de recursos; sin olvidar, como se apuntaba más arriba, los cambios en las pautas distributivas que se deducen.

\section{CUADRO 7}

Las industrias de consumo antes y después de la Guerra Civil $(1958=100)$

\begin{tabular}{|c|c|c|c|}
\hline & Alimentación & Textil & Otras \\
\hline 1935 & 105,5 & 78,8 & 49,8 \\
\hline 1940 & 67,0 & 57,7 & 32,3 \\
\hline
\end{tabular}

FUENTE: Elaboración propia.

IV

El nuevo índice de la producción industrial española de la posguerra civil, sin diferir excesivamente del IPIES, rebaja las tasas de crecimiento del período y trata de calibrar con mayor rigor el impacto de la guerra. Quizá su mayor ventaja consista en que, al ser presentado de forma desagregada, permite plantear la industrialización en términos de los cambios en la composición del producto. De esta forma puede observarse la magnitud del cambio intraindustrial que, a pesar del estancamiento relativo de los años cuarenta, es constante desde mediados de la década. Y, por otra parte, se pone de manifiesto cómo la intensa intervención estatal acelera este proceso a costa de la capacidad de compra de los asalariados. 


\section{APENDICE}

\section{La construcción del índice}

A partir de la teoría sobre números índices y teniendo en cuenta las limitaciones en cuanto a la información disponible, los índices que se presentan en la tabla número 1 obedecen a la siguiente fórmula:

$$
I_{i}^{s}=\frac{\sum_{j}^{m_{s}} p_{t j} q_{i j}}{\sum_{j}^{m_{s}} p_{t j} q_{t j}} \times 100
$$

donde $q$ son las cantidades producidas; $p$ son los valores añadidos unitarios; $J=1 \ldots m_{s}$ son los productos considerados; $m_{s}$ es el número de productos incluidos en cada sector; $\mathrm{s}=1 \ldots 7$ son los distintos sectores (energía, $\mathrm{m}_{1}=8$; extracción y primera transformación de minerales no energético, $\mathrm{m}_{2}=26$; química, $\mathrm{m}_{3}=14$; transformados metálicos, $\mathrm{m}_{4}=7$; industrias alimentarias, $\mathrm{m}_{5}=13$; industria textil, $\mathrm{m}_{6}=9$, y otras industrias manufactureras, $\left.\mathrm{m}_{7}=6\right) ; j=1940$...t., es el año a que corresponde el índice, y $t$ es el año base de ponderación, o sea, 1958 en este caso.

Dado que para el período 1940-58 el IPIES usa las ponderaciones de 1958, la fórmula es idéntica, con la diferencia de que aquí se aplica a los productos de cada sector para obtener siete índices sectoriales, mientras que en el IPIES se aplica al conjunto de la industria para obtener un único índice.

\section{TABLA 1}

Los indices sectoriales

\begin{tabular}{llccccccc}
\hline & Energia & Mineria & Quimica & Metal & Aliment. & Textil & Otras \\
\hline 1940 & $\ldots \ldots \ldots \ldots \ldots \ldots \ldots \ldots$ & 33,70 & 43,07 & 15,98 & 38,46 & 66,97 & 57,70 & 32,30 \\
1941 & $\ldots \ldots \ldots \ldots \ldots \ldots \ldots \ldots \ldots$ & 33,68 & 39,53 & 15,85 & 32,37 & 63,92 & 53,27 & 33,10 \\
1942 & $\ldots \ldots \ldots \ldots \ldots \ldots \ldots \ldots \ldots$ & 37,35 & 40,52 & 16,93 & 33,56 & 72,52 & 58,61 & 32,97 \\
1943 & $\ldots \ldots \ldots \ldots \ldots \ldots \ldots \ldots$ & 39,16 & 43,25 & 16,61 & 36,01 & 72,75 & 60,79 & 33,53 \\
1944 & $\ldots \ldots \ldots \ldots \ldots \ldots \ldots \ldots \ldots$ & 41,35 & 39,96 & 19,68 & 33,31 & 79,14 & 71,88 & 38,24 \\
1945 & $\ldots \ldots \ldots \ldots \ldots \ldots \ldots \ldots \ldots$ & 40,62 & 37,52 & 18,69 & 31,95 & 70,81 & 69,04 & 34,31 \\
1946 & $\ldots \ldots \ldots \ldots \ldots \ldots \ldots \ldots$ & 44,86 & 43,21 & 26,53 & 39,28 & 68,51 & 70,06 & 37,14 \\
1947 & $\ldots \ldots \ldots \ldots \ldots \ldots \ldots \ldots \ldots$ & 46,16 & 41,10 & 27,68 & 35,83 & 84,95 & 62,89 & 43,80 \\
1948 & $\ldots \ldots \ldots \ldots \ldots \ldots \ldots \ldots$ & 47,36 & 42,99 & 31,33 & 39,55 & 83,52 & 62,20 & 39,81 \\
1949 & $\ldots \ldots \ldots \ldots \ldots \ldots \ldots \ldots \ldots$ & 46,50 & 46,95 & 33,93 & 41,47 & 69,22 & 61,03 & 39,92
\end{tabular}




\begin{tabular}{llrrrrrrr}
\hline & Energia & Mineria & Quimica & Metal & Aliment. & Textil & Otras \\
\hline $1950 \ldots \ldots \ldots \ldots \ldots \ldots \ldots \ldots$ & 51,81 & 53,53 & 42,80 & 46,38 & 76,30 & 58,30 & 43,30 \\
1951 & $\ldots \ldots \ldots \ldots \ldots \ldots \ldots \ldots$ & 57,62 & 56,12 & 48,03 & 46,15 & 73,40 & 62,88 & 44,12 \\
1952 & $\ldots \ldots \ldots \ldots \ldots \ldots \ldots \ldots$ & 64,09 & 64,76 & 56,75 & 56,56 & 98,75 & 71,14 & 53,07 \\
1953 & $\ldots \ldots \ldots \ldots \ldots \ldots \ldots \ldots$ & 63,41 & 64,71 & 56,86 & 55,76 & 92,28 & 78,37 & 55,67 \\
1954 & $\ldots \ldots \ldots \ldots \ldots \ldots \ldots \ldots \ldots$ & 69,27 & 72,20 & 66,09 & 65,23 & 87,66 & 79,41 & 66,41 \\
1955 & $\ldots \ldots \ldots \ldots \ldots \ldots \ldots \ldots \ldots$ & 74,05 & 79,40 & 77,84 & 71,40 & 87,05 & 83,76 & 79,28 \\
1956 & $\ldots \ldots \ldots \ldots \ldots \ldots \ldots \ldots$ & 81,62 & 82,49 & 84,72 & 80,06 & 97,02 & 89,27 & 85,90 \\
1957 & $\ldots \ldots \ldots \ldots \ldots \ldots \ldots \ldots \ldots$ & 91,07 & 86,99 & 92,07 & 87,51 & 99,73 & 94,75 & 96,40 \\
1958 & $\ldots \ldots \ldots \ldots \ldots \ldots \ldots \ldots$ & 100,00 & 100,00 & 100,00 & 100,00 & 100,00 & 100,00 & 100,00 \\
\hline VAB & $\ldots \ldots \ldots \ldots \ldots \ldots \ldots \ldots$ & 22764,7 & 21627,6 & 12015,4 & 31710,5 & 27633,7 & 25215,9 & 20225,2 \\
G. Cobert $\ldots \ldots \ldots \ldots \ldots \ldots$. & 0,92 & 0,68 & 0,33 & 0,92 & 0,41 & 0,58 & 0,23 \\
\hline
\end{tabular}

FUENTE: Elaboración propia a partir de Carreras (1983) y TIOE-58 (1962), según los métodos explicados en el texto.

\section{TABLA 2}

Aportación de cada sector

\begin{tabular}{|c|c|c|c|c|c|c|c|}
\hline & & Energía & Mineria & Quimica & Metal & Construc. & $I P I E \cdot P G C$ \\
\hline 1940 & $\cdots$ & 4,76 & 5,78 & 1,19 & 7,57 & 24,56 & 43,85 \\
\hline 1941 & 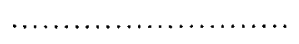 & 4,76 & 5,30 & 1,18 & 6,37 & 23,44 & 41,05 \\
\hline 1942 & $\ldots \ldots \ldots \ldots \ldots \ldots \ldots \ldots$ & 5,27 & 5,44 & 1,26 & 6,60 & 25,74 & 44,31 \\
\hline 1943 & $\ldots$ & 5,53 & 5,80 & 1,24 & 7,08 & 26,19 & 45,84 \\
\hline 1944 & $\ldots \ldots$ & 5,84 & 5,36 & 1,47 & 6,55 & 29,61 & 48,83 \\
\hline 1945 & .... & 5,74 & 5,03 & 1,39 & 6,29 & 27,24 & 45,69 \\
\hline 1946 & $\cdots$ & 6,34 & 5,80 & 1,98 & 7,73 & 27,36 & 49,20 \\
\hline 1947 & $\ldots$ & 6,52 & 5,51 & 2,06 & 7,05 & 29,90 & 51,04 \\
\hline 1948 & $\ldots$ & 6,69 & 5,77 & 2,34 & 7,78 & 29,04 & 51,61 \\
\hline 1949 & $\ldots \ldots$ & 6,57 & 6,30 & 2,53 & 8,16 & 26,42 & 49,98 \\
\hline 1950 & & 7,32 & 7,18 & 3,19 & 9,12 & 27,63 & 54,45 \\
\hline 1951 & n.t., & 8,14 & 7,53 & 3,58 & 9,08 & 27,96 & 56,28 \\
\hline 1952 & $\ldots \ldots \ldots \ldots \ldots \ldots \ldots \ldots$ & 9,05 & 8,69 & 4,23 & 11,13 & 34,72 & 67,81 \\
\hline 1953 & ............. & 8,96 & 8,68 & 4,24 & 10,97 & 35,06 & 67,91 \\
\hline 1954 & $\cdots \cdots \cdot$ & 9,78 & 9,69 & 4,93 & 12,83 & 35,78 & 73,01 \\
\hline 1955 & ...... & 10,46 & 10,65 & 5,80 & 14,05 & 37,97 & 78,93 \\
\hline 1956 & $\ldots \ldots \ldots \ldots \ldots \ldots \ldots \ldots \ldots \ldots$ & 11,53 & 11,07 & 6,32 & 15,75 & 41,37 & 86,03 \\
\hline 1957 & 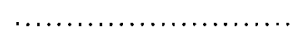 & 12,86 & 11,67 & 6,86 & 17,22 & 44,01 & 92,63 \\
\hline 1958 & $\ldots \ldots \ldots \ldots \ldots \ldots \ldots \ldots \ldots$ & 14,12 & 13,42 & 7,45 & 19,67 & 45,33 & 100,00 \\
\hline
\end{tabular}

FUENTE: Elaboración propia a partir de la tabla número 1 (véase nota 22). 
Para la construcción del índice agregado, se calcula el valor añadido sectorial implícito en cada índice:

$$
\mathrm{VAB}_{i}^{s}=\frac{\sum_{j}^{m_{s}} p_{t j} q_{i j}}{\sum_{j}^{m_{s}} p_{t j} q_{t j} / \mathrm{VAB}_{t}^{s}}
$$

La suma de los valores añadidos sectoriales para cada año respecto al valor añadido total generado por la industria en 1958 constituye la aproximación a la evolución del producto industrial que aquí se propone:

$$
\mathrm{IPIE} \cdot \mathrm{PGC}_{i}=\frac{\sum_{s=1}^{7} \mathrm{VAB}_{i}^{s}}{\sum_{s=1}^{7} \mathrm{VAB}_{t}^{s}} \times 100^{22}
$$

La tabla número 2 presenta este índice desglosado en términos de la aportación de cada sector.

21 Donde el denominador son los grados de cobertura que aparecen en la última línea de la tabla número 1. Por tanto, el supuesto que se está imponiendo es la constancia en el grado de cobertura para cada sector:

$$
\text { G. Cob.s }=\frac{\Sigma_{j} p_{i j} q_{i j}}{\mathrm{VAB}_{i}^{s}}, \quad \text { para todo } i
$$

22 Nótese que esto equivale a calcular la media de los indices sectoriales ponderada por la aportación de cada uno de ellos al valor añadido industrial en el año de ponderación:

$$
I_{i}=\frac{\Sigma_{s} \mathrm{VAB}_{i}^{s}}{\Sigma_{s} \mathrm{VAB}_{t}^{s}} \times 100=\Sigma_{s}\left[\frac{\Sigma_{j} p_{t j} q_{i j}}{\Sigma_{j} p_{t j} q_{t j}} \mathrm{VAB}_{t}^{s}\right] \frac{100}{\overline{\Sigma_{s} \mathrm{VAB}_{t}^{s}}}
$$

Dado que $\Sigma_{s} \mathrm{VAB}_{t}^{s}$ es una constante (el valor añadido industrial de 1958):

$$
I_{i}=\Sigma_{s}\left[\frac{\Sigma_{j} p_{t j} q_{i j}}{\Sigma_{j} p_{t j} q_{t j}} \times 100 \frac{\mathrm{VAB}_{t}^{s}}{\Sigma_{s} \mathrm{VAB}_{t}^{s}}\right]=\Sigma_{s} l_{i}^{s} \frac{\mathrm{VAB}_{t}^{s}}{\Sigma_{s} \mathrm{VAB}_{t}^{s}}
$$




\section{BIBLIOGRAFIA}

AlCAIDE, Julio (1976): «Una revisión urgente de la serie de renta nacional española en el siglo XX», en Datos básicos para la bistoria financiera de España, 1850-1975, vol. I, Madrid, Instituto de Estudios Fiscales, pp. 1127-1150.

Anuario Estadistico de España, Madrid, varias instituciones y varios años.

BARCIELA LópEZ, C. (1981): "El estraperlo de trigo en la postguerra», en Moneda y crédito, n. 161, pp. 17-37.

- (1986): «Introducción. Segunda parte; los costes del franquismo en el sector agrario: ruptura del proceso de transformaciones», en R. GARRABOU, C. BARCIELA, y J. I. JIMÉNEZ BlANCO (eds.): Historia agraria de la España contemporánea. Vol. III. El fin de la agricultura tradicional (1900-1960), Barcelona, Crítica.

BrañA, F. J.; Buesa, M., y MOLERo, J. (1979): "El fin de la etapa nacionalista: industrialización y dependencia en España, 1951-1959", en Investigacioines Económicas, n. 9, pp. 151-207.

-_- (1984): El Estado y el cambio tecnológico en la industrialiqación tardia. Un análisis del caso español, Madrid, Fondo de Cultura Económica.

BUESA, M. (1984): «Las restricciones a la libertad de industria en la política industrial española (1938-1963)», en Información Comercial Española, n. 606, pp. 107-121.

CARRERAS, Albert (1983): La producció industrial espanyola i italiana des de mitjan segle XIX fins a l'actualitat, Bellaterra, tesis doctoral (inédita), presentada en la Universidad Autónoma de Barcelona.

(1984): «La producción industrial española, 1842-1981: construcción de un indice anual», en Revista de Historia Económica, n. 1, pp. 127-157.

(1987): "La industria: atraso y modernización", en J. NADAL, A. CARRERAS y C. SUDRIA (eds.): La economia española en el siglo XX. Una perspectiva bistórica, Barcelona, Ariel, pp. 280-312.

(ed.) (1989, a): Estadisticas bistóricas de España (siglos XIX-XX), Madrid, Fundación Banco Exterior.

- (1989, b): «Depresión económica y cambio estructural durante el decenio bélico (1936-1945)», en J. L. GARCIA DELGADO (ed.): El primer franquismo. España durante la segunda guerra mundial, Madrid, Siglo XXI, pp. 3-33.

CATALÁN, Jordi $(1989$ a): «Autarquía y desarrollo de la industria de fábrica durante la segunda guerra mundial. Un enfoque comparativo», en J. L. GARCía Delgado (ed.): El primer franquismo. España durante la segunda guerra mundial, Madrid, Siglo XXI, pp. 35-88.

(1989, b): «Reconstrucción y desarrollo industrial. Tres economías del sur de Europa, 1944-1953», comunicación presentada al IV Congreso de Historia Económica, Alicante.

Claver A, Juan (1976): "El estraperlo en los años cuarenta», en Información Comercial Española, junio, pp. 91-97.

Clavera, J.; Esteban, J. M.; Mones, M. A.; Montserrat, A., y Ros, J. (1973): Capitalismo español: de la autarquia a la estabilización (1939-1959), 2 vols., Madrid, Edicusa.

CONSEjo DE ECONOMía NACIONAI. (1965): La renta nacional de España, 1949-1964, Madrid. 
Doncies, Juergen B. (1976): La industrialización en España. Politicas, logros, perspectivas, Barcelona, Oikos-Tau.

FARRi-EsCOFET, E. (1980): «Inflación y bloqueo de la inversión (1939-1953)», en Banca Catalana, n. 57, pp. 1-21.

FEnOAlteA, S. (1976): "Real Value Added and the Measurement of Industrial Production», en Annals of Economic and Social Measurement, 1, Pp. 111-137.

Fontana, J., y NAdAL, J.: «España, 1914-1970» (1980), en C. M. Cipoli.A: Historia Económica de Europa, vol. 6. Economias contemporáneas, Barcelona, Ariel, t. 2, pp. 95-163.

GAMIR, L.: «El período 1939-1959: la autarquia y la política de estabilización» (1972), en L. GAmir (ed.): Politica Económica de España, Madrid, Ed. Guadiana, pp. 1330.

Garcia Delgado, J. L. (1985): «Notas sobre el intervencionismo económico del primer franquismo", en Revista de Historia Económica, III, 1.

(1986): "Estancamiento industrial e intervencionismo económico durante el primer franquismo», en J. FONTANA (ed.): España bajo el franquismo, Barcelona, Crítica, pp. 170-191.

(1987): «La industrialización y el desarrollo económico de España durante el franquismo", en J. NADAL, A. CARRERAS y C. SUdRIA (eds.): La economía española en el siglo XX. Una perspectiva bistórica, Barcelona, Ariel, pp. 164-189.

GonZÁLEZ, M. J. (1979): La economia politica del franquismo, 1940-1970. Dirigismo, mercado y planificación, Madrid, Tecnos.

Gonzalez POR'Tlla, M. (1989): «El País Vasco en la posguerra: crecimiento económico y especialización industrial», en J. L. García Delgado (ed.): El primer franquismo. España durante la segunda guerra mundial, Madrid, Siglo XXI, pp. 89-102.

-- y Garmendia, J. M. (1988): La posguerra en el Pais Vasco. Politica, acumulación, miseria, Donostia, Kriselu.

HOFFMANN, W. G. (1958): The Growth of Industrial Economies, Manchester University Press.

Instituto Nacional de Estadística (1982): Numeros indices de la producción industrial, monografía técnica, Madrid.

Molinero, C., e Ysas, P. (1985): "Patria, justicia y pan», en Nivells de vida $i$ condicions de treball a Catalunya, 1939-1951, Barcelona, La Magrana.

Montserrat, A., y Ros Hombravelia, J. (1972): "Entorn del retard en el recobrament dels nivells macroeconòmics de pre-guerra a l'Espanya dels 1950», en Recerques, n. 2, pp. 221-246.

NAREDO, J. M. (1989): "Crítica y revisión de las series históricas de renta nacional en la postguerra», comunicación presentada al IV Congreso de Historia Económica, Alicante.

Paris Egull.Az, Higinio (1981): Cincuenta años de economia española, 1930-1980, Madrid, J. Sánchez de Ocaña.

PRADOS DE LA ESCOSURA, Leandro (1988): De imperio a nación, crecimiento y atraso económico en España (1780-1930), Madrid, Alianza.

RIBAS, Albert (1978): L'economia catalana sota el franquisme (1939-1953), Barcelona, Ediciones 62 . 
SCOTT, I. O. (1952): "The Gerschenkron Hypothesis of Index Number Bias", en Review of Economics and Statistics, noviembre, pp. 386-387.

SUDRIA, Carles (1987): «Un factor determinante: la energía», en J. NADAL, A. CARReras y C. SUDRIA (eds.): La economia española en el siglo XX. Una perspectiva bistórica, Barcelona, Ariel, pp. 313-363.

Tabla «input-output» de la economia española. Año 1958 (1962), Madrid, Organización Sindical.

Usher, D. (1980): The Measurement of Economic Growth, Nueva York, Columbia University Press. 